\title{
AVULSÃO DO PLEXO BRAQUIAL EM CÃES - 3. ELETRONEUROESTIMULAÇÃO DOS NERVOS RADIAL, MEDIANO, ULNAR E MUSCÜLOCUTÂNEO. ${ }^{1}$
}

\section{BRACHIAL PLEXUS AVULSION IN DOGS - 3. ELECTRONEUROSTIMULATION OF RADIAL, MEDIAN, ULNAR AND MUSCULOCUTANEOUS NERVES}

\author{
Mônica Vicky Bahr Árias² Angelo João Stopiglia ${ }^{3}$
}

\section{RESUMO}

\begin{abstract}
O objetivo deste trabalho foi relacionar os aspectos clínicos, neurológicos e histopatológicos (descritos nas partes le 2 deste trabalho) com os resultados obtidos após estimulação elétrica dos nervos radiai, mediano, ulnar e musculocutâneo. Realizou-se a estimulação elétrica destes nervos durante o ato cirúrgico no qual foram coletados os fascículos para histopatolo gia. Os nervos radial, mediano e ulnar de todos os cães submetidos à eletroneuroestimulação apresentaram evidências de degenera- ção. enquanto que o nervo musculocutâneo apresentava função próxima do normal em $25 \%$ dos casos. A associação dos resultados do exame neurológico, da histologia e da eletroneuroestimulação sugeriu envolvimento quase que total das raízes do plexo braquial, enfatizando a necessidade de continuidade de pesquisas na área, visando principalmente a recuperação das raizes nervosas envolvidas.
\end{abstract}

Palavras-chave: avulsâo do plexo braquial, cães, sistema nervoso periférico, eletroneuroestimulação.

\section{SUMMARY}

The purpose ofthis work was to relate lhe clinicai, neurological and histopathotogical aspects (as described in the sections I and 2 ofthis work) with the obtained results after the electric stimulation of radial, median, ulnar and musculocutaneous nerrves. The electric stimulation of these nerves was realized during the cirurgic act, when the fascicle were obtained for the histopathologic examination. The radial, median and ulnar nerves of ali dogs submitted to electroneurostimulation presented evidences of degeneration, while the musculocutaneous nerve present almost normal functions in $25 \%$ of the cases. The interpretation ofthe results obtained from neurologic, histologic and electroneurostimulation examination suggested the almost total involvement of brachial plexus in ali cases. This work emphasized the need for further research in this área with the main purpose of recuperating the involved roots.

Key words: brachial plexus avulsion, dogs, peripheral nervous system, electroneurostimulation.

\section{INTRODUÇÃO}

A avulsâo do plexo braquial é neuropatia de ordem traumática, decorrente de tração do membro, que pode causar lesão das raízes nervosas, de seus ramos ventrais e do próprio plexo braquial (SUMMERS et al., 1995).

De modo geral testes eletrodiagnósticos são úteis no estudo de distúrbios envolvendo a unidade motora (corpo celular, axônio, junção neuromuscular e fibras musculares). Por serem capazes de avaliar somente a função das estruturas envolvidas, para se atingir o diagnóstico etiológico é necessária a correlação com o histórico, exame

\footnotetext{
${ }^{1}$ Parte da tese apresentada pelo primeiro autor como requisito parcial para obtenção do título de Mestre junto ao Departamento de Cirurgia da Faculdade de Medicina Veterinária e Zootecnia (FMVZ), Universidade de São Paulo (USP). Apoio bolsa de estudos d FAPESP ${ }_{2}^{2}$ Médico Veterinário, Professor Assitente, Centro de Ciências Biológicas, Universidade Estadual de Londrina, Caixa Postal 6001, 86051970 - Londrina, PR. Autor para correspondência.

${ }^{3}$ Médico Veterinário, Professor Associado do Departamento de Cirurgia, FMVZ, USP.
} 
físico e outros dados laboratoriais (LIEBERMAN, 1982).

ALLAM et al. (1949), utilizando aparelho de estimulação elétrica percutânea, avaliaram casos clínicos de paralisia do membro torácico em cães orientando a conduta terapêutica de acordo com a resposta obtida após estimulação dos nervos radial, ulnar e mediano. O método utilizado se baseava na ocorrência de contração muscular após estímulo elétrico, nos casos de regeneração neuronal. O nervo em processo de degeneração ou em regeneração se torna menos sensível que o normal requerendo intensidade maior de corrente elétrica para deflagrar resposta motora.

Após lesão aguda ao nervo, devido a ocorrência de poucas mudanças no tecido nervoso, não são observadas alterações nos testes eletrodiagnósticos quando o nervo é estimulado distalmente ao local de lesão. Após 3 a 4 dias, tem início a degeneração axonal. Somente 8 a 21 dias após a lesão é que se inicia o aparecimento de alterações eletromiográfícas e de velocidade de condução nervosa. Se este prazo não for respeitado, estes exames terão valor limitado na avaliação das lesões do plexo braquial (LIEBERMAN, 1982).

Imediatamente após a avulsão, antes da degeneração walleriana atingir segmentos mais distais, as fibras motoras podem conduzir o impulso evocado em uma velocidade dentro do normal sendo que após a ocorrência da degeneração walleriana, os impulsos não podem ser conduzidos (SHORES, 1993). Os anestésicos e tranquilizantes administrados não influenciam as respostas motoras induzidas eletricamente, no entanto suprimem a resposta sensorial, que forneceria informações suplementares (BOWEN, 1978).

Segundo KLINE \& NULSEN (1982), estímulo de baixa intensidade deve ser aplicado ao tronco nervoso através de duas agulhas hipodérmicas separadas por $1 \mathrm{~cm}$. Um resultado negativo deve ser reavaliado pela recolocação das agulhas. A voltagem fornecida deve ser suficiente para evocar a contração muscular. Para evitar que ocorra resposta devido ao estímulo de nervos adjacentes intactos, o nervo a ser examinado deve ser separado do tecido ao redor. A ausência de resposta ocorre imediatamente após o trauma nos pontos proximais à lesão. Se o estímulo não deflagra resposta motora, há forte evidência de lesão completa ou ausência de regeneração. A presença de alguns axônios em regeneração pode permitir resposta positiva. Nos casos de denervação, pode ocorrer também contração muscular lenta, considerada reação de degeneração.

Para correta avaliação da resposta motora obtida, é necessário lembrar que o nervo radial $\left(\mathrm{C}_{7^{-}}\right.$ $\mathrm{T}_{1}$ ) é motor para todos os músculos extensores do cotovelo, carpo e dedos, inervando os músculos tríceps braquial, extensor radial do carpo, ulnar lateral, extensor digital comum e extensor digital lateral; o nervo musculocutâneo $\left(\mathrm{C}_{6}-\mathrm{C}_{8}\right)$ inerva os músculos bíceps braquial, braquial e coracobraquial; o nervo mediano $\left(\mathrm{C}_{8}-\mathrm{T}_{2}\right)$ supre os músculos flexor digital superficial e flexor do carpo radial e o nervo ulnar $\left(\mathrm{C}_{8}-\mathrm{T}_{2}\right)$ é responsável pela inervação dos músculos flexor do carpo ulnar e flexor digital profundo (KNECHT \& RAFFE, 1985, SHORES, 1993).

Este trabalho teve como objetivo avaliar a resposta motora após estimulação elétrica dos nervos radial, mediano, ulnar e musculocutâneo relacionando estes resultados aos aspectos clínicos, neurológicos e histopatológicos de cães com avulsão do plexo braquial, descritos nas partes 1 e 2 desta pesquisa.

\section{MATERIAIS E MÉTODOS}

Para realização dos testes eletrodiagnósticos construiu-se um aparelho de eletroneuroestimulação a partir de quatro baterias pequenas de 1,5 Volts (V), conectadas a um dispositivo que permitia selecionar a voltagem entre 0,5 e 6,0 V. Através de uma extensão o aparelho foi conectado a um par de agulhas de aço inoxidável. A extensão foi mantida esterilizada através da colocação em recipiente com pastilhas de paraformaldeído e as agulhas foram submetidas à esterilização em estufa.

$$
\text { Para padronização }
$$

eletroneuroestimulação utilizou-se um cão normal anestesiado com clorpromazina, tiopental e halotano. Para realização da parte clínica/cirúrgica, foram utilizados 8 cães de ambos os sexos, sendo cinco fêmeas e três machos. com idade variando entre 6 meses e 11 anos, trazidos ao Hospital Veterinário da Faculdade de Medicina Veterinária e Zootecnia da Universidade de São Paulo por apresentarem quadro clínico compatível com avulsão das raízes do plexo braquial. Os cães foram submetidos a exame clínico rotineiro e exame neurológico completo.

Durante o ato cirúrgico, antes da coleta de cada fascículo para biópsia, procedeu-se à eletroneuroestimulação dos nervos radial, mediano, ulnar e musculocutâneo, respeitando-se o prazo mínimo de três semanas entre a ocorrência da avulsão e a realização deste exame.

Os nervos foram isolados do tecido 
adjacente com o uso de uma fita de látex estéril, e estimulados inicialmente com $0,5 \mathrm{~V}$, colocando-se os eletrodos em contato com o tecido nervoso e mantendo-se $1,0 \mathrm{~cm}$ de distância entre os dois poios. O resultado negativo era reavaliado aumentando-se gradativamente o estímulo, até $6,0 \mathrm{~V}$ e caso não houvesse resposta motora, procedia-se a mudança de posição dos eletrodos. Padronizou-se como resposta motora típica de degeneração quando a contração muscular estivesse ausente ou fraca (BOWEN, 1978), sob estímulo maior ou igual do que 3,0 Volts, quando comparada com cão normal.

\section{RESULTADOS}

A realização deste teste em cão normal mostrou resposta motora evidente após estímulo com $0,5 \mathrm{~V}$, sendo que o estímulo do nervo radial levou à extensão do cotovelo e carpo, o estímulo do nervo musculocutâneo levou à flexão do cotovelo, e o estímulo dos nervos mediano e ulnar levou à flexão do carpo e dígitos.

Em 93,8\% dos nervos submetidos à eletroneuroestimulação observou-se resposta muscular ausente ou lenta, realizando-se o exame em média dois meses após a ocorrência da lesão (Tabela 1). O estímulo dos nervos radial, mediano e ulnar evidenciou este tipo de resposta em todos os casos e em seis casos
(75\%) após estímulo do nervo musculocutâneo. Observou-se resposta próxima do normal (1,O V) após estímulo deste mesmo nervo em dois (25\%) dos casos. Enquanto sempre houve leve resposta contrátil à estimulação do nervo radial com voltagens iguais ou superiores a $3 \mathrm{~V}$, não houve nenhuma contração muscular após estímulo máximo $(6, \mathrm{OV})$ dos nervos mediano e ulnar em quatro cães, e após estímulo máximo do nervo musculocutâneo em dois cães.

Em todos os oito animais com perda dos reflexos tricipital, extensor do carpo radial, atrofia dos músculos inervados pelo nervo radial $\mathrm{e}$ incapacidade de estender e apoiar o membro no solo havia, ao realizar-se a eletroneuroestimulação deste nervo, resposta compatível com degeneração nervosa.

Em seis pacientes com ulceração ou abrasão da face dorsal da mão e anestesia cutânea nos dermátomos relacionados aos nervos mediano e ulnar, observou-se resposta muscular ausente ou lenta após eletroneuroestimulação destes nervos .

Em dois cães com capacidade de flexão do cotovelo e presença parcial do reflexo interdigital, observou-se resposta próxima do normal $(1,0 \mathrm{~V})$ após eletroneuroestimulação do nervo musculocutâneo e resposta muscular lenta em dois cães com capacidade parcial de flexão do cotovelo. Em quatro cães com incapacidade de flexão do cotovelo e atrofia dos músculos inervados pelo nervo musculocutâneo havia, à eletroneuroestimulação deste nervo, resposta muscular ausente ou lenta.

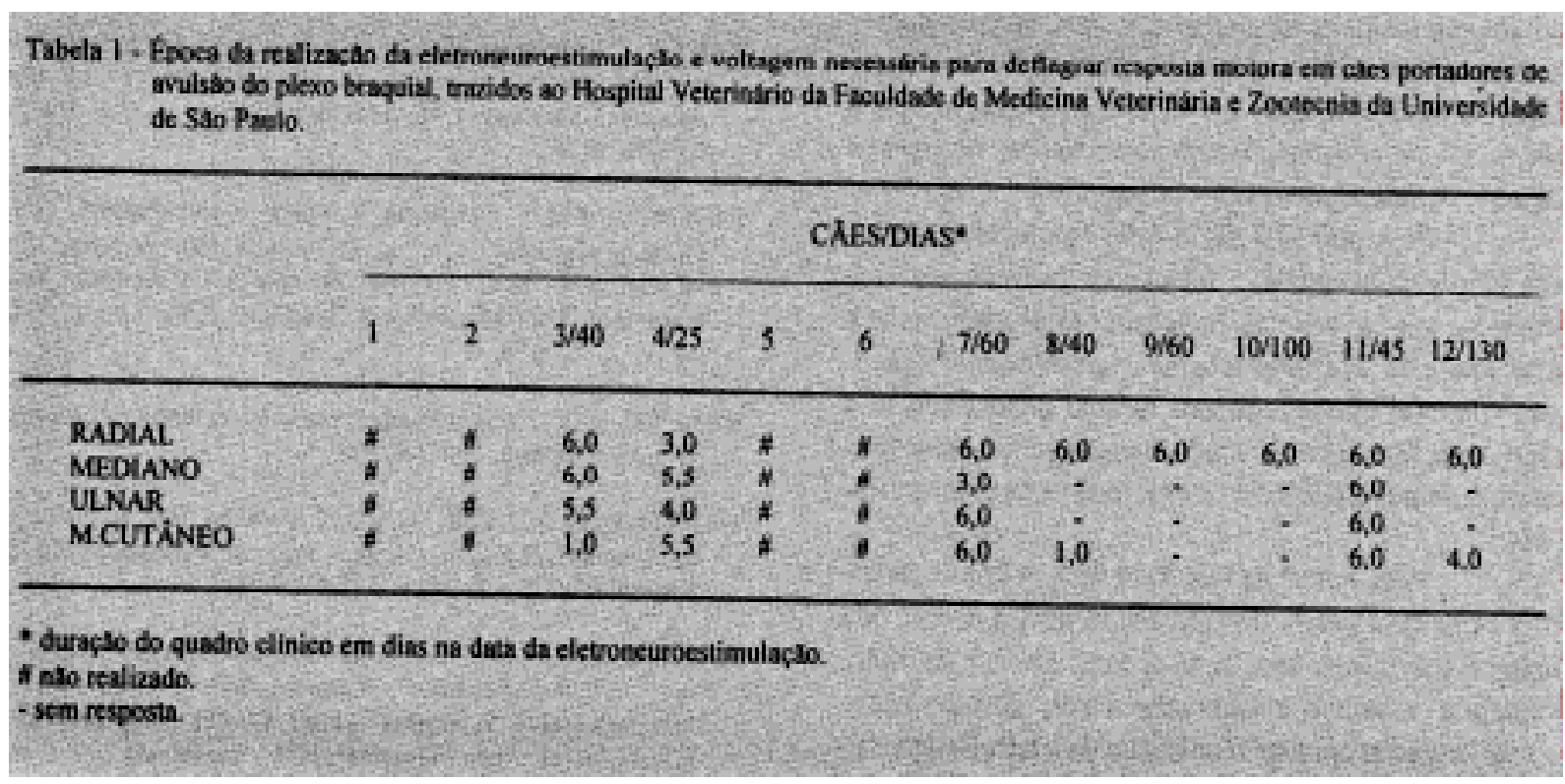

Ciência Rural, v. 27n. 11997 


\section{DISCUSSÃO}

Segundo BOWEN (1978), um nervo lesado pode reter a sensibilidade por três a 21 dias, dependendo da distância entre o local da lesão e o músculo alvo. Após este tempo a resposta do músculo denervado se toma lenta ou ausente, resposta encontrada na maioria dos nervos examinados. $\mathrm{O}$ intervalo entre a ocorrência da lesão e a realização do exame foi suficiente para evitar respostas falso positivas, como comprovado por resultados histológicos (ARIAS et al, 1997), mesmo sendo este tempo insuficiente para que ocorresse regeneração nervosa.

Segundo SIMPSON et al. (1985), quase todos os animais com avulsão do plexo braquial apresentam envolvimento dos nervos radial, mediano e ulnar, resultado semelhante ao obtido neste estudo, onde observou-se em todos os cães submetidos à eletroneuroestimulação lesão destes nervos. Para este mesmo autor, o nervo musculocutâneo está frequentemente mas nem sempre envolvido, aspecto também observado neste trabalho, onde observou-se lesão parcial deste nervo em $25 \%$ dos casos. Estes resultados são semelhantes aos obtidos por STEIMBERG (1979), que utilizando eletrodiagnóstico observou menor comprometimento do nervo musculocutâneo, quando comparado com os nervos radial, mediano e ulnar, indicando que a porção caudal do plexo braquial é mais afetada do que a porção cranial.

Segundo BRAUND (1994) a atrofia dos músculos extensores do membro e ausência dos reflexos extensor do carpo radial e tricipital são aspectos importantes em pacientes com lesão do nervo radial. Estas alterações foram encontradas em todos os cães e a lesão deste nervo foi confirmada posteriormente durante a eletroneuroestimulação, mostrando a confiabilidade do exame neurológico para este nervo, ao contrário do que ocorre com os nervos musculocutâneo, mediano e ulnar.

Segundo SHORES (1993), nas lesões caudais do plexo braquial, a função do nervo musculocutâneo é apenas parcial, havendo em; alguns casos a capacidade de flexionar total ou parcialmente o cotovelo. Isto foi observado em quatro casos, sendo a lesão deste nervo confirmada apenas pela eletroneuroestimulação e posteriormente pelo exame histológico.

Para KNECHT \& RAFFE (1985) e SHORES (1993), a perda da função do nervo ulnar, que fornece inervação sensorial à face lateral da mão, predispõe o animal à ulceração acral. Comparandose este aspecto com os resultados da eletroneuroestimulação, foi constatado que o nervo ulnar estava de- generado em todos os casos, justificando assim a presença desta lesão, apesar dos cuidados dispensados aos pacientes.

Segundo GRIFFITHS et al. (1974) e BAILEY (1984) a falta de padronização dos dermátomos em cães dificulta a avaliação de áreas de anestesia cutânea. Segundo SHORES (1993), a detecção da integridade do nervo mediano depende principalmente da avaliação da sensibilidade cutânea, que em alguns casos, segundo BAILEY (1984), pode ser difícil de interpretar devido à maneira como alguns cães respondem aos estímulos dolorosos. Neste estudo, a eletroneuroestimulação demonstrou facilmente a lesão dos nervos mediano e ulnar em todos os cães examinados, sendo o resultado mais confiável do que o exame neurológico. A indicação de técnicas cirúrgicas como panartrodese do carpo ou transposição de tendões nas avulsões parciais, segundo KNECHT \& RAFFE (1985) teria melhores resultados se a função dos nervos mediano, ulnar e musculocutâneo pudesse ser testada previamente. Isto poderia ser verificado através do método de estimulação elétrica de nervos realizado no presente trabalho.

\section{REFERÊNCIAS BIBLIOGRÁFICAS}

ALLAM, M.W., NULSEN, F.E., LEWEY, F.H. Electrical intraneural bipolar stimulation of peripheral nerves in the dog. Journal of the American Veterinary Medicai Association, v. 114, p. $87-9,1949$.

ÁRIAS, M.V.B., BRACARENSE, A.P.F.L., STOPIGLIA, A.J. Avulsão do plexo braquial em cães - 2. Biópsia fascicular e histologia dos nervos radial, mediano, ulnar e museulocutâneo. Ciência Rural,v. 27, n. 1, p. 81-85, 1977.

BAILEY, C.S. Patterns ofcutaneous anesthesia associated with braehial plexus avulsions in the dog Journal of the American Veterinary Medicai Association, v. 185, n. 8, p. 889-99, 1984.

BOWEN, J.M. Peripheral nerve electrodiagnostics, electromyography, and nerve conduction velocity. In: HOERLEIN, B.F. Canine neurology. Diagnosis and treatment. 3. ed. Philadelphia, Saunders, 1978, 3 ed, p. 25479.

BRAUND, K.G. Clinical syndromes in veterinary neurology. St. Louis: Mosby, 1994. 477 p.

GRIFFITHS, D.G., DUNCAN, I.D., LAWSON, D.D. Avulsion of the braehial plexus-2. Clinicai aspects. Journal of Small Animal Practice, v. 15, p. 177-82, 1974.

KLINE, D.G., NULSEN, F.E. Acute injuries of peripheral nerves. In: YOUMANS.J.R. Neurological surgery. A comprehensive reference guide to the diagnosis and management of neurosurgical problems 2 . ed. Philadelphia: Saunders,, 1982, p. 2362-429. 
KNECHT, C.D., RAFFE, M.R. Diseases ofthe brachial plexus. In: NEWTON, C.D., NUNAMAKER, D.M. Textbook of small animal orthopaedics. Philadelphia: Lippincott, 1985, p. 817-23.

LIEBERMAN, J.S. Neuromuscular electrodiagnosis. In. YOUMANS, J.R. Neurological surgery. A comprehensive reference guide to the diagnosis and management of neurosurgical problems 2. ed. Philadelphia: Saunders, 1982, p. 617-35.

SHORES, A. Traumatic and neoplastic diseases ofthe brachial plexus. In: BOJRAB, M.J. Diseases mechanism in small animais. 2. ed. Philadelphia: Lea\& Febiger, 1993, p. 1175-82.

SIMPSON, S.T., KORNEGAY, J.N., RAFFE, M.R. Surgical diseases ofp.eripheral nerves. In : SLATTER, D.H. Textbook of small animal surgery. Philadelphia, WB Saunders, 1985, p.1335-8

STEIMBERG, H.S. The use ofelectrodiagnostic techniques in evaluating traumatic brachial plexus root injuries, Journal of the American Animal Hospital Association. 15:621, 1979.

SUMMERS, B.A., CUMMINGS, J.F., DE LAHUNTA, A. Veterinary neuropathology. St Louis: Mosby, 1995, 527 p. 